\title{
The global rotation of solar activity structures
}

\author{
D. Heristchi and Z. Mouradian
}

Observatoire de Paris, LESIA, 92195 Meudon, France

e-mail: [Djamshid.Heristchi ; Zadig. Mouradian]@obspm. fr

Received 15 February 2008 / Accepted 16 January 2009

\begin{abstract}
Aims. This paper investigates the rotation rate of solar activity from a global point of view, considering daily index or flux, instead of individual structures. It determines the rotation rate variations inside the cycle by two-year sequences and the comprehensive study of the cycle.

Methods. The method consists of harmonic analysis of series of daily monitoring of solar activity. One is the sunspot number covering cycles 9 to 23 (over 157 years) and the second the flux of the corona at $2800 \mathrm{MHz}$ from cycle 19 to 25 (over 53 years). These long series were analysed by the maximum entropy method for the measurement of rotation frequency.

Results. The findings displayed in this paper are: i) sunspots and coronal flux show the same global rotation rates; ii) the solar rotation unfolding during the cycle is complex, without any systematic pattern; iii) the analysis of cyclic solar activity rotation leads to variation of the rotation rate with a period of 52.4 years; iv) the present result supports the existence of high-level cycles made of five eleven-year cycles; the global rotation rate also has this periodicity; v) no asymmetry was found in the northern and southern hemisphere rotation rates.
\end{abstract}

Key words. Sun: rotation - Sun: sunspots - Sun: corona - Sun: activity

\section{Introduction}

Since the basic work of Carrington on solar rotation, studies have been performed the framework of differential rotation. Two methods are mainly used: the tracer method and the spectroscopic method. The classic review papers of Howard (1984), Schröter (1985), Libbrecht \& Morrow (1991), Snodgrass (1992) and Beck (1999) compare and discuss the results of different measurements.

In order to reach a more synthetic view of solar rotation, we suggest the method of global rotation applied to structures of solar activity. The measurement of solar global rotation uses series of daily observations of structures of the same nature over a determined time interval. The principle is to employ an existing series of daily amounts of fluxes or numbers of structures located over the solar disk, regardless of their position or state of evolution. These daily data are analysed to obtain the most frequent rotation rate in the considered time interval. The global method revealed some new characteristic aspects of solar activity. Of course this method, as with the two aforementioned methods, has its limit, but each one partially contributes to solar physics. The global method was proposed by Mouradian et al. (2000, 2002) and Mouradian \& Heristchi (2005).

In the next section we describe the basic characteristics of the global method. The next two sections (Sects. 3 and 4) display the data used in the present paper and the method of analysis. Section 5 gives the results for two types of measurements of rotation rate (RR), one in sequences of two years (730 days) all along the cycle, and the second one for the whole cycle. Then the Carrington RR and the solar activity per hemispheres are calculated with the global method. In Sect. 6 we discuss the results.

\section{The global method for the measure of solar activity rotation rate}

In the present study we use already existing long series of observations of sunspot number and the coronal radio flux at $10.7 \mathrm{~cm}$. Because these two types of structures characterise the solar activity, the determined rotation rate is that of activity (essentially of magnetic flux) independent of quiet sun rotation. The analysis of data is performed either in two-year sequences $(730 \mathrm{~d})$ covering the cycle or for the whole cycle. The use of the global method averages out the fine structures, local layout and short time evolution. Individual structures, local proper motions, meridian drift or differential rotation are analysed together inside the considered time interval. These measurements are naturally weighted by flux intensity or number of considered elements.

All activity structures used were created by the action of magnetic field, the method thus is based on the rotation of regions with highest magnetic flux (Gaizauskas et al. 1983; Castenmiller et al. 1986). It emphasizes frequencies of the return time of structures (Balthasar 2007) providing a measure of the rotation period. The global rotation point of view is somewhat related to the recurrent sunspots method used by Newton \& Nunn (1951).

We note that the global rotation measurement can be applied under three limiting conditions: i) the studied structures must show a non uniform distribution in longitude, their clustering strengthens the non uniformity (Balthasar 2007; Usoskin et al. 2007; Berdyugina \& Usoskin 2003; Gilman \& Howard 1984; Trellis 1971), ii) the lifetime of structures or clusters has to be longer than the solar rotation period (Pojoga \& Cudnik 2002), so that their successive passage over the disk produces pulses of the recorded quantity and iii) the considered structures must 
have a spectral or morphological specific character, which can be detected.

The global method shows the following characteristics:

- the proposed method is free of the possible errors of the Carrington coordinate system (Schröter 1985);

- it has relatively low sensitivity to the seeing conditions during the observations;

- the global method performed over a complete terrestrial revolution avoids the effect of variation of orbital parameters shorter than one year (Graf 1974; Juckett 2000);

- this method can determine the RR of past solar activity;

- the results are not influenced by the layout of structures;

- no foreshortening effect;

- it does not take into account the latitude variation of structures, such as the differential rotation, or proper motion, or meridian drift;

- if the analysis does not concern the full cycle, then the possible effect of differential rotation may affect the results.

\section{The data}

We performed two series of observations, namely sunspot number (cycles 9 to 23, from 1843 to 2006) and coronal radio flux at $10.7 \mathrm{~cm}$ (cycles 19 to 23, from 1953 to 2006). These are two solar indexes of different natures, different altitudes, different spatial resolutions and different techniques of observation, but they belong to the same solar component: the active region.

- The classic sunspot number data is collected and archived by the solar influences data analysis centre (SIDC) of the Royal Observatory of Belgium. Daily sunspot number over the solar disk is an average of a high number of observations distributed all around the world. Since 1992, SIDC has collected sunspot numbers for northern and southern hemispheres as well as the whole disk data. The time resolution of SIDC data is one day.

- The second series of observations is the daily radio flux recordings at $10.7 \mathrm{~cm}(2800 \mathrm{MHz})$ by the dominion radio astrophysical observatory (DRAO) of the Herzberg Institute of Astrophysics in Canada. From there the corrected Series D at local midday is extracted. Radio flux at $10.7 \mathrm{~cm}$ concerns full disk radiation flux, out side of any transient event and it is the most characteristic physical index of solar active regions. The effective height for the generation of $10.7 \mathrm{~cm}$ radio emission is between 15000 and $60000 \mathrm{~km}$ above the photosphere (Mouradian et al. 2002), therefore in the low corona. In a few cases of gaps in the data, we interpolated between the closest values.

- The comparison of northern and southern hemisphere rotation is studied by using the data of sunspots (1945 to 2004) obtained by assembling the observations from the Kanzelhöhe Observatory in Austria with those from the Astronomical Institute in Tatranská Lomnica, in Slovakia (Temmer et al. 2006). The sum of both hemispheres is normalized to the corresponding sunspot number of full disk data from SIDC. This data is hereafter referred to as K\&SP.

In order to determine the period of sunspot rotation with high accuracy, continuous daily data is required. Before 1849.5 sunspot tables show a high number of gaps (Fig. 1 of Hoyt \& Schatten 1998), so that the power spectra cannot be correctly computed by MEM (Balthasar 2007). Consequently the RR of cycle 9 was calculated only with the end of cycle, i.e. sequences 4 to 6 .

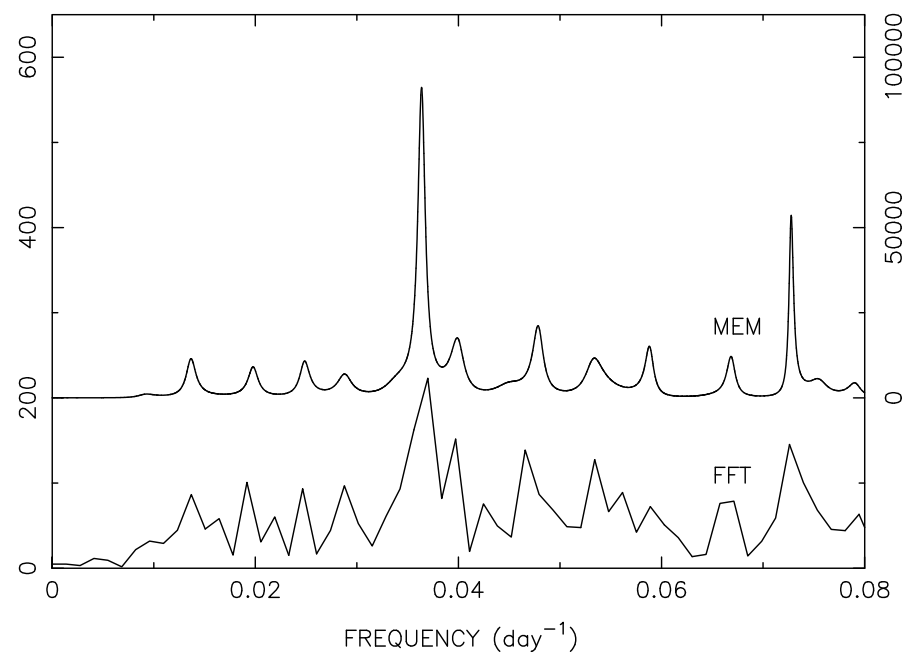

Fig. 1. The power spectrum of sunspot number over 730 days (4 September 1981 to 4 September 1983). The upper curve shows the power spectrum obtained by the maximum entropy method (MEM), the scale is on the right hand side. Bottom, the spectrum by a fast Fourier transform (FFT) of the same period, its scale is on the left hand side.

Similarly, large gaps in data were noticed in radio flux lists before 1953.

The recorded solar activity indexes (sunspot number and radiation flux) can be split into two parts. One runs all along the whole cycle of activity and shows the general shape of the cycle. The other one, added to the previous part, is an irregular periodic fluctuation due to the passage of active structures over the solar disk. This is the slow varying component free of any transient event (flares, sudden disappearance of filaments, surges etc.) initially erased by data centres. It will be the basic material for our harmonic analysis.

\section{Method of analysis}

To prepare the data for frequency analysis, we had to extract through filtering the slow varying component of the solar disk. First we scanned the recordings with a triangular normalised window of 81 days, which supplies a smooth function. To reveal the fluctuations the smooth function is subtracted from the recordings in order to point out an alternate function around a straight line. This is the clear slow varying component of solar activity, that will be treated by harmonic analysis (Mouradian et al. 2002). For each considered time interval, the position of the straight line was corrected to obtain the sum of alternate functions equal to zero.

The analysis of data was performed using the fast Fourier transform (FFT) and the maximum entropy method (MEM). We systematically applied both methods for optimum control. The power spectra provided by MEM gives higher spectral resolution than FFT; we therefore decided to use only MEM with 4001 frequencies in the considered spectral range. All values given in tables and figures are from MEM. Figure 1 shows both power spectra (in the frequency range 0 to $0.08 \mathrm{~d}^{-1}$ ) of the same sunspot sample. The obtained power spectra are composed of a few peaks (Fig. 6) and sometimes only one is in the considered spectral range (Fig. 1). Each peak is characterized by the frequency of maximum amplitude and by energy (average of frequencies weighted by amplitudes). These figures are always identical or, in the case of a blend, very close. In some ambiguous cases (two close peaks showing almost the same energy) we chose the one in agreement with the previous and the following sequences. 
From the frequency of the peak are successively determined the synodic period, the sidereal period (Wittmann 1996) and the solar RR in units of degree per day $(\% / d)$.

The errors contained in observational material from the data centre cannot be determined. The analysis method may generate errors due to two sources: a) power spectrum analysis provides inaccurate frequencies; b) the frequency and amplitude inhomogeneities of solar phenomena.

a) In order to determine the errors introduced by the method of computation, we composed a number of synthetic series of "observations" with two to five periodic curves and analysed their frequencies by MEM. The difference between given and computed frequencies is the error of computed frequences $\pm 0.015^{\circ} / \mathrm{d}$.

b) The measure of the second type of error is made by testing the real data: the influence introduced by sequence length $(730,730+5 \%$ and $730+10 \%$ days $)$ and by the shift in time of $730 \mathrm{~d}$ with 36 and with $73 \mathrm{~d}$. The error in both cases is the difference between initial and displaced sequences. This tests gives an error of $\pm 0.05 \%$ d. Consequently we took this figure as representative of errors of calculated frequences.

\section{Results}

The above-mentioned analysis was applied to the data of solar activity. We display the results obtained by: 730 day sequences, the full cycles, the Carrington period and the comparison of northern and southern hemisphere asymmetry.

\subsection{Two year analysis}

Each cycle was covered by an entire number of contiguous sequences of $730 \mathrm{~d}$, keeping in mind the variation of RR during the cycle. We first placed a two-year sequence on the maximum of activity, then two other sequences backward toward the beginning of the cycle and 2 to 4 forward, till the next minimum. Consequently the maximum is always situated at sequence 3 . Table 1 contains the sequences of sunspots and of coronal radio flux rotation rates of each cycle. The RR of different sequences does not show regular evolution inside the cycle, as is expected from the differential rotation rule.

\subsubsection{Sunspot and corona rotation rate comparison}

In this study, the first question we investigated was whether the rotation rates of various sorts of structures in the solar atmosphere were different, as some published analyses of power spectra suggest (Mouradian 2007). In order to answer this question, the records of sunspot numbers and corona radio flux were used. Figure 2 and Table 1 give their RR by sequences of two years, which are in good agreement. The standard deviation between the two series is $\mathrm{SD}=0.19^{\circ} / \mathrm{d}$. It is obvious that, even if the observation technique and nature of structures are different, the same method of analysis gives very similar results. The similarity of RR variation inside the cycles of both series shows that these variations cannot be qualified as noise, but they are really due to movements in the solar atmosphere. In Sect. 5.2 we show that the RRs of the full cycle (Fig. 5 and Table 2) give identical figures for sunspots and corona rotation in the limits of the determined error bars.

The similarity of RRs of two series of data is also a strong argument for the accuracy of the method of analysis. The sunspot
Table 1. Sidereal sunspot and coronal rotation rates for two year sequences of each cycle. For estimated error see Sect. 4.

\begin{tabular}{|c|c|c|c|c|}
\hline Cycle & Seq. & Beginning & $\operatorname{SIDC}\left({ }^{\circ} / \mathrm{d}\right)$ & DRAO $(\% / d)$ \\
\hline 9 & 1 & 1843.455 & $\ldots$ & $\ldots$ \\
\hline 9 & 2 & 1845.452 & $\ldots$ & $\ldots$ \\
\hline 9 & 3 & 1847.452 & & $\ldots$ \\
\hline 9 & 4 & 1849.449 & 14.02 & $\ldots$ \\
\hline 9 & 5 & 1851.449 & 14.38 & $\ldots$ \\
\hline 9 & 6 & 1853.447 & 14.12 & $\ldots$ \\
\hline 10 & 1 & 1855.455 & 13.64 & $\ldots$ \\
\hline 10 & 2 & 1857.452 & 14.40 & $\ldots$ \\
\hline 10 & 3 & 1859.452 & 12.93 & $\ldots$ \\
\hline 10 & 4 & 1861.449 & 13.68 & $\ldots$ \\
\hline 10 & 5 & 1863.449 & 13.91 & $\ldots$ \\
\hline 11 & 1 & 1866.126 & 14.15 & $\ldots$ \\
\hline 11 & 2 & 1867.126 & 14.75 & $\ldots$ \\
\hline 11 & 3 & 1870.123 & 15.42 & $\ldots$ \\
\hline 11 & 4 & 1872.123 & 14.40 & $\ldots$ \\
\hline 11 & 5 & 1874.120 & 13.34 & $\ldots$ \\
\hline 11 & 6 & 1876.121 & 14.04 & $\ldots$ \\
\hline 11 & 7 & 1878.117 & 14.25 & $\ldots$ \\
\hline 12 & 1 & 1879.795 & 14.22 & $\ldots$ \\
\hline 12 & 2 & 1881.792 & 13.35 & $\ldots$ \\
\hline 12 & 3 & 1883.792 & 13.72 & $\ldots$ \\
\hline 12 & 4 & 1885.789 & 14.48 & $\ldots$ \\
\hline 12 & 5 & 1887.789 & 14.64 & $\ldots$ \\
\hline 13 & 1 & 1888.874 & 14.02 & $\ldots$ \\
\hline 13 & 2 & 1890.874 & 13.74 & $\ldots$ \\
\hline 13 & 3 & 1892.872 & 13.34 & $\ldots$ \\
\hline 13 & 4 & 1894.871 & 14.25 & $\ldots$ \\
\hline 13 & 5 & 1896.869 & 14.65 & $\ldots$ \\
\hline 13 & 6 & 1898.868 & 14.47 & $\ldots$ \\
\hline 13 & 7 & 1900.869 & 14.80 & $\ldots$ \\
\hline 14 & 1 & 1901.671 & 14.58 & $\ldots$ \\
\hline 14 & 2 & 1903.671 & 14.63 & $\ldots$ \\
\hline 14 & 3 & 1905.668 & 13.27 & $\ldots$ \\
\hline 14 & 4 & 1907.668 & 13.33 & $\ldots$ \\
\hline 14 & 5 & 1909.665 & 14.26 & $\ldots$ \\
\hline 15 & 1 & 1912.586 & 14.91 & $\ldots$ \\
\hline 15 & 2 & 1914.586 & 13.85 & $\ldots$ \\
\hline 15 & 3 & 1916.583 & 14.16 & $\ldots$ \\
\hline 15 & 4 & 1918.583 & 14.50 & $\ldots$ \\
\hline 15 & 5 & 1920.580 & 14.18 & $\ldots$ \\
\hline 16 & 1 & 1922.838 & 13.92 & $\ldots$ \\
\hline 16 & 2 & 1924.835 & 13.98 & $\ldots$ \\
\hline 16 & 3 & 1926.835 & 14.77 & $\ldots$ \\
\hline 16 & 4 & 1928.832 & 15.01 & $\ldots$ \\
\hline 16 & 5 & 1930.832 & 13.84 & $\ldots$ \\
\hline 17 & 1 & 1933.003 & 14.62 & $\ldots$ \\
\hline 17 & 2 & 1935.003 & 13.80 & $\ldots$ \\
\hline 17 & 3 & 1936.999 & 14.12 & $\ldots$ \\
\hline 17 & 4 & 1938.999 & 15.05 & $\ldots$ \\
\hline 17 & 5 & 1940.997 & 14.69 & $\ldots$ \\
\hline 17 & 6 & 1942.997 & 14.01 & $\ldots$ \\
\hline 18 & 1 & 1944.003 & 14.86 & $\ldots$ \\
\hline 18 & 2 & 1945.999 & 14.83 & $\ldots$ \\
\hline 18 & 3 & 1947.999 & 14.01 & $\ldots$ \\
\hline 18 & 4 & 1949.997 & 14.31 & $\ldots$ \\
\hline 18 & 5 & 1951.997 & 14.90 & \\
\hline 19 & 1 & 1953.452 & 14.20 & 14.38 \\
\hline 19 & 2 & 1955.452 & 14.16 & 14.33 \\
\hline 19 & 3 & 1957.449 & 14.08 & 14.43 \\
\hline 19 & 4 & 1959.449 & 13.72 & 13.89 \\
\hline 19 & 5 & 1961.446 & 13.76 & 13.88 \\
\hline 20 & 1 & 1964.361 & 13.64 & 14.08 \\
\hline 20 & 2 & 1966.361 & 13.83 & 13.90 \\
\hline 20 & 3 & 1968.359 & 14.07 & 14.04 \\
\hline 20 & 4 & 1970.359 & 14.89 & 15.07 \\
\hline
\end{tabular}


Table 1. continued.

\begin{tabular}{rrrrr}
\hline \hline Cycle & Seq. & Beginning & SIDC $\left({ }^{\circ} / \mathrm{d}\right)$ & DRAO $\left(^{\circ} / \mathrm{d}\right)$ \\
\hline 20 & 5 & 1972.356 & 14.08 & 14.26 \\
20 & 6 & 1974.356 & 15.24 & 15.24 \\
21 & 1 & 1975.784 & 14.31 & 14.24 \\
21 & 2 & 1977.682 & 14.73 & 14.69 \\
21 & 3 & 1979.682 & 13.98 & 13.94 \\
21 & 4 & 1981.679 & 14.00 & 14.18 \\
21 & 5 & 1983.679 & 14.05 & 14.18 \\
22 & 1 & 1984.942 & 14.53 & 14.54 \\
22 & 2 & 1986.942 & 14.04 & 13.95 \\
22 & 3 & 1988.939 & 14.59 & 14.71 \\
22 & 4 & 1990.939 & 14.52 & 14.56 \\
22 & 5 & 1992.936 & 14.15 & 14.27 \\
22 & 6 & 1994.936 & 14.75 & 14.96 \\
23 & 1 & 1996.115 & 14.36 & 14.39 \\
23 & 2 & 1998.112 & 13.95 & 13.82 \\
23 & 3 & 2000.112 & 14.90 & 14.66 \\
23 & 4 & 2002.110 & 14.67 & 14.53 \\
23 & 5 & 2004.112 & 14.41 & 14.51 \\
\hline
\end{tabular}

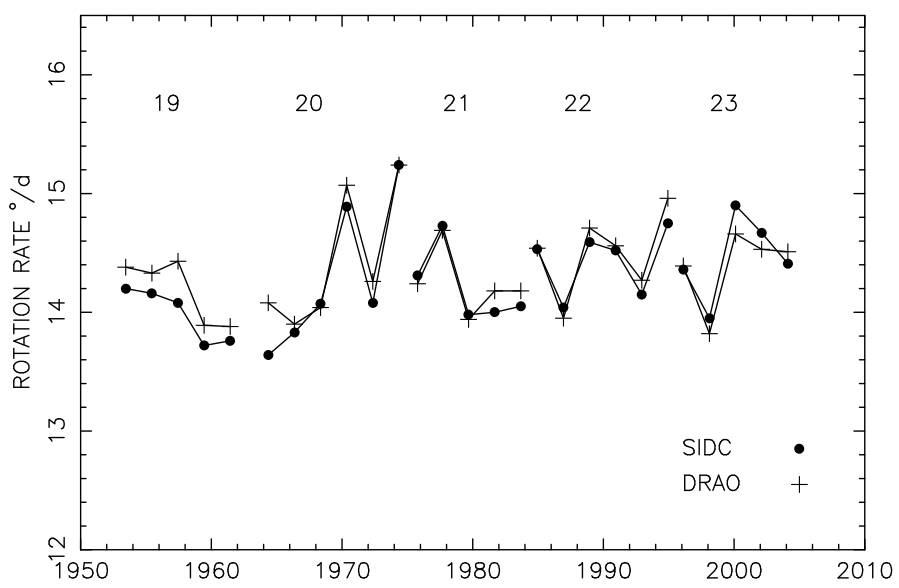

Fig. 2. Sidereal rotation rates for sequences of 730 days (cycles 19 to 23) of the sunspots number (SIDC) and of the coronal radio flux at $10.7 \mathrm{~cm}$ (DRAO). The upper row indicates the number of cycles. Dots of each cycle are join together and plotted in the middle of the sequence.

and corona data reveal that the entire atmosphere of active regions rotates rigidly, in spite of a number of transient events and magnetic field disruptions.

\subsubsection{Time variation of rotation rate}

The second property displayed in Fig. 2, the general increase of the RR of cycles, is also mentioned by other authors (Howard 1984). Our results confirm this general trend. The continuous increase cannot be a constant slope along time but rather must necessarily be a periodic variation or a randomly obtained variation. To answer this unambiguously, the studied time period was extended backward, first till the end of the century, then until 1843.

The cycles in Fig. 3 can be grouped into three series: the earlier section during the 19th century (cycles 9 to 13) and the two other sections formed by cycles 14 to 18 and 19 to 23 . All three series show the RR acceleration for each epoch of 5 cycles. First these sections were fitted by a straight line using the least squares method. This gives us the slopes: $0.005( \pm 0.007), 0.010( \pm 0.006)$ and $0.011( \pm 0.005)^{\circ} / \mathrm{d}$ per set of five cycles. The time variation of RR given in Fig. 3 highlights three time periods, each lasting about 5 cycles ( $\sim 50$ years).

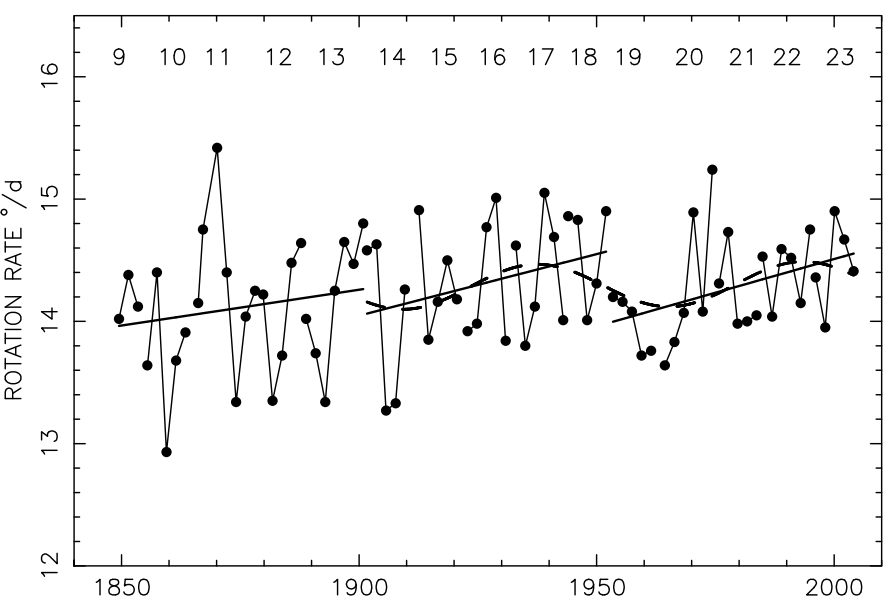

Fig. 3. Variation of the sidereal rotation rates of $730 \mathrm{~d}$ sequences (cycles 9 to 23) of the sunspot number. The position on the $x$-axis of rotation rate is at the midpoint sequence. The straight lines are the regression lines of the three series of cycles. The dashed curve represents the periodic function of $55 \mathrm{y}$ fitted over cycles 14 to 23 (see Sect. 5.2).

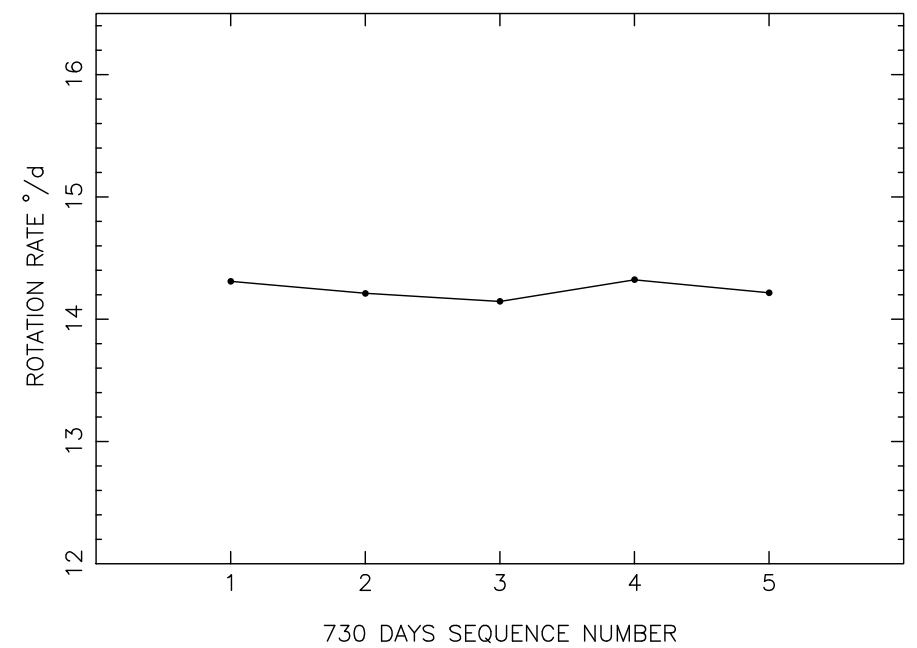

Fig. 4. The superimposed epoch analyses of 14 cycles sequence by sequence. Sequence 3 corresponds to the maximum of solar activity.

We mention that the RR at the maximum of cycle activity (sequence 3 ) does not show any special behaviour.

\subsubsection{Rotation rate variation during the cycle}

As can be seen in Figs. 2 and 3, the RRs have variable behaviour for each cycle. Because the two series of data of different structures and independent measurements produced similar results, we were led to consider that the variations of RR during the cycle are mainly of physical origin. To check this hypothesis we carried out a superimposed epoch analysis of sequences of 730 days by averaging the RR of the 14 cycles (10 to 23 ) in Fig. 4 . We found a constant figure within $\mathrm{SD}=0.45^{\circ}$ d (i.e. $\sim 3 \%$ ) for the first 5 analysed sequences, which proves the random character of these velocity fields. This assertion will be confirmed in Sect. 5.4 and discussed in Sect. 6.

\subsection{Rotation rate of full cycle}

The complete global rotation analysis is carried on using the full cycle rotation rate. It is expected that the full cycle analysis provides a more accurate measurement than the two-year 


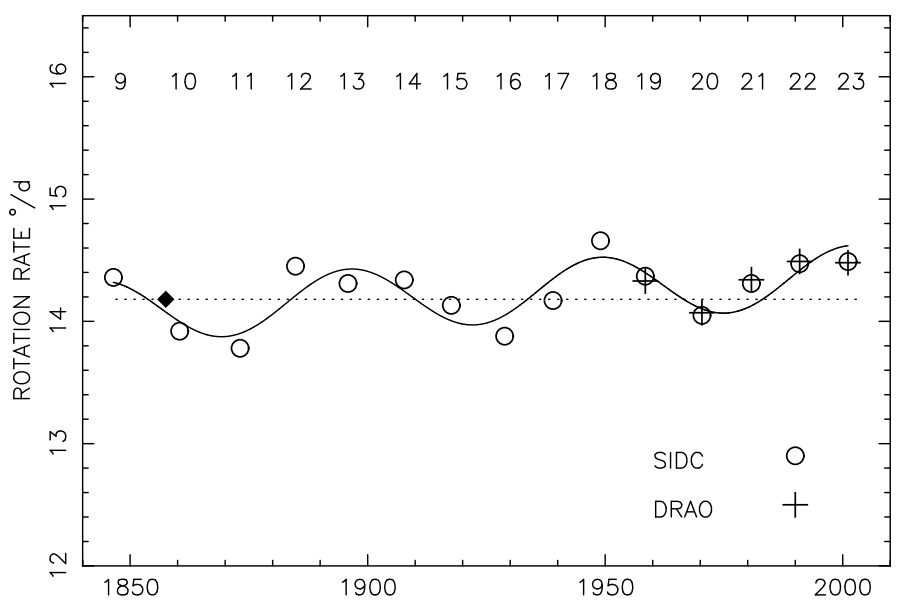

Fig. 5. Full cycle rotation rate of sunspots and coronal radio flux. The position of dots on the $x$-axis is that of the mid cycle. The fitted function shows a period of $52.4 \mathrm{y}$ to which a general positive gradient is added. The black diamond and dotted line show the Carrington rotation rate.

Table 2. Sidereal rotation rates of full cycles 9 to 23. For estimated error see Sect. 4

\begin{tabular}{rrrrr}
\hline \hline Cycle & Beginning & Duration $(\mathrm{d})$ & SIDC $\left({ }^{\circ} / \mathrm{d}\right)$ & DRAO $\left(^{\circ} / \mathrm{d}\right)$ \\
\hline 9 & 1849.449 & 2190 & 14.36 & $\ldots$ \\
10 & 1855.455 & 3650 & 13.92 & $\ldots$ \\
11 & 1866.126 & 5110 & 13.78 & $\ldots$ \\
12 & 1879.795 & 3650 & 14.45 & $\ldots$ \\
13 & 1888.874 & 5110 & 14.31 & $\ldots$ \\
14 & 1901.671 & 4380 & 14.34 & $\ldots$ \\
15 & 1912.586 & 3650 & 14.13 & $\ldots$ \\
16 & 1922.838 & 4380 & 13.88 & $\ldots$ \\
17 & 1933.003 & 4380 & 14.17 & $\ldots$ \\
18 & 1944.003 & 3650 & 14.66 & $\ldots$ \\
19 & 1953.452 & 3650 & 14.37 & 14.33 \\
20 & 1964.361 & 4380 & 14.05 & 14.07 \\
21 & 1975.784 & 3650 & 14.31 & 14.34 \\
22 & 1984.942 & 4380 & 14.47 & 14.49 \\
23 & 1996.115 & 3650 & 14.49 & 14.48 \\
\hline
\end{tabular}

sequences, because it brings out the basic and permanent frequencies, instead of temporal intense rotation structures. Also, it erases the effect of differential rotation and the fine structures of active regions over the length of the cycle, providing a single figure for solar rotation rate per cycle. It is obvious that the average per cycle of two-year sequences is not necessarily equal to the whole cycle rotation rate.

In Table 2 and Fig. 5 we display the temporal variation of RR of the full solar cycle of sunspots (9 to 23) and of coronal radio flux (19 to 23). The whole cycle analysis was performed over the entire number of 730 day sequences, covering all data used in Sect. 5.1. The 15 full cycles of sunspots and 5 cycles of corona clearly show a periodic variation of the RR. We note that even cycles 9 to 13, which in Fig. 3 appear as noisy, are here in good agreement with the other cycles. The time variation of cyclic RRs is fitted by a periodic function and by a general gradient.

$$
\begin{aligned}
\Omega_{\circ / \mathrm{d}}= & 0.26( \pm 0.06) \times \sin \left[\frac{2 \pi}{52.4( \pm 2.3)} \times t+1.47( \pm 0.50)\right] \\
& +0.0017( \pm 0.0009) \times t+14.09( \pm 0.09)
\end{aligned}
$$

where $t$ is the time starting from 1840 and in parenthesis are indicated the root mean square error of computed parameters. The

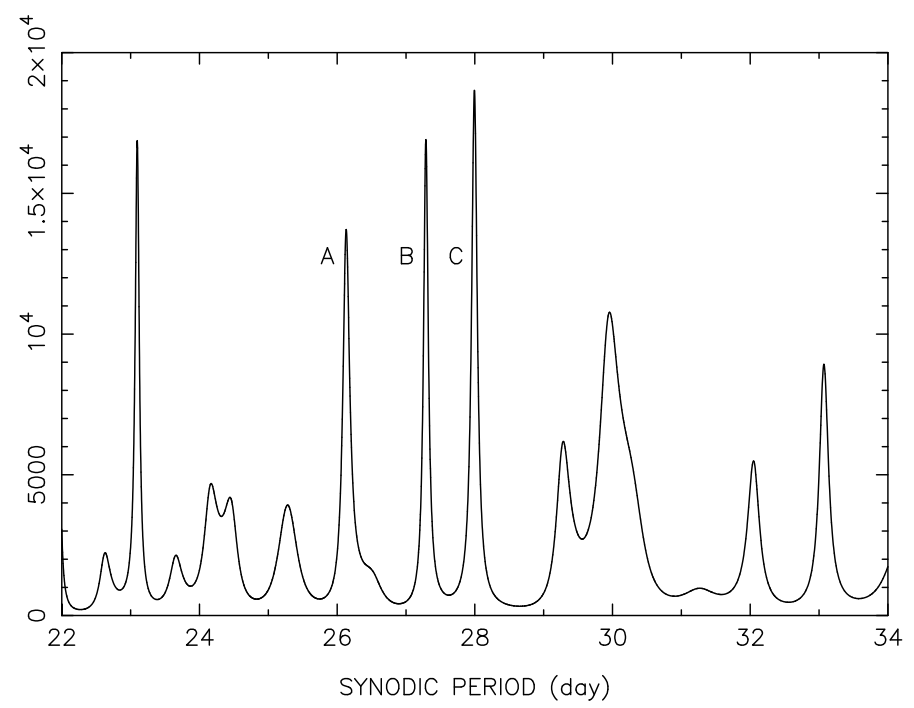

Fig. 6. Power spectrum of Carrington period 1853 to 1861 . The three revealed sidereal rotation rates correspond to: $A=14.78, B=14.18$ (the Carrington Rate) and $C=13.87^{\circ} / \mathrm{d}$.

standard deviation of measured $\mathrm{RR}$ in regard to the $\Omega$ function is $\mathrm{SD}= \pm 0.13^{\circ} / \mathrm{d}( \pm 0.9 \%)$. The observational material includes errors in measurement which remain unknown and therefore cannot be corrected. Their effect appears in Fig. 5. The general gradient shows an increase of $1.2 \%$ per century and the RR variations due to periodicity can reach $3.7 \%$. The 52.4 y periodicity corresponds to a rotational frequency of $0.60 \mathrm{nHz}$.

We attempted to see if a periodicity of five cycles also be detected with $730 \mathrm{~d}$ sequences. This was done using a slightly different sine curve with a periodicity of 55( \pm 11$)$ years and an amplitude of $0.18( \pm 0.09)^{\circ} / \mathrm{d}$ (dashed curve in Fig. 3) to which was added a linear increasing gradient of $0.0017( \pm 0.0009)^{\circ} / \mathrm{d} / \mathrm{y}$. The fit was carried out on cycle 14 to 23 . Including cycle 9-13 gives some unreliable parameters, certainly due to the high level of noise. Consequently, the two slopes of cycles 14-18 and 1923 are a first estimate for the $\sim 50$ y periodic variation of the solar activity rotation (see Sect. 5.2). Figure 3 shows how close the slopes and the sine functions are in some periods.

\subsection{Carrington rotation rate}

The second half of the 19th century includes the well-known period used by Carrington to measure the solar rotation rate and to define the Carrington coordinate system. This was measured over 2692 days (7.37 y), starting during the end phase of the 19th cycle until the early decreasing phase of the 10th cycle (9 November 1853 to 24 March 1861). Within this period, Carrington observed the 5290 sunspots in 954 groups.

We determine the RR by using the global method and SIDC data corresponding exactly to that time interval. The power spectrum of Fig. 6 shows three peaks at synodic periods: $A=26.14$, $B=27.27$ and $C=27.98 \mathrm{~d}$. Peak B corresponds to that determined by Carrington i.e. the sidereal RR of $14.18^{\circ} / \mathrm{d}$. If the current computation respects the epoch and extent of old data, then the old and new data are not identical. Carrington uses single sunspot positions, whereas we use daily Wolf number; also the techniques of computation are not the same, the MEM has higher spectral resolution that may reveal side frequencies. 


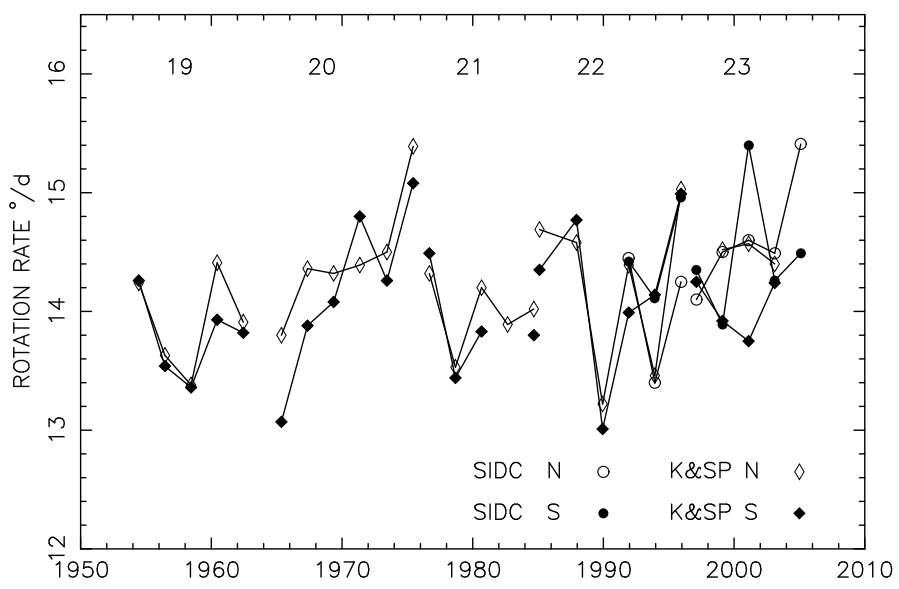

Fig. 7. Northern and southern hemisphere rotation rate of $730 \mathrm{~d}$ sequences of cycles 19 to 23 .

In Fig. 5 the measured "Carrington rate" is depicted by a diamond and a dotted line showing the constant value in the last 150 years.

\subsection{Northern and southern hemisphere rotation}

In Fig. 7, the RRs of $730 \mathrm{~d}$ sequences of the northern and of the southern solar hemispheres are displayed. We use the data from K\&SP (cycles 19 to 23) and SIDC (decreasing phase of the cycle 22 and the cycle 23). Neither systematic differences nor important discrepancies can be seen between RRs of the hemispheres; they are just a little noisier compared to disk data (Fig. 3). Here also a general increase of RR similar for both hemispheres during the five cycles can be noticed. The slope of linear regression for northern and southern hemispheres are respectively $0.0084( \pm 0.0071)$ and $0.0075( \pm 0.0073)^{\circ} / \mathrm{d} / \mathrm{y}$, quite close to that given in the case of the whole disk $0.011( \pm 0.005)^{\circ} / \mathrm{d} / \mathrm{y}$. The same slope is present in Fig. 3 this allows us to suppose that a periodic variation of 52 y may be the cause. The RR variations are similar for both hemispheres, showing that these are due to real changes of a physical nature and not to noise in the measurements or method.

Cycle 23 is the only entire cycle which can be analysed with both SIDC and K\&SP data. The obtained RR for northern and southern hemispheres respectively are: 14.61 and $14.50^{\circ} / \mathrm{d}$ for SIDC and 14.57 and $14.51^{\circ}$ d for K\&SP, in good agreement in the limits of errors. This guarantees the quality of K\&SP data. The full disk RR of cycle 23 of SIDC is 14.49 (Table 2). The asymmetry of sunspot numbers between both hemispheres was established for instance by Balester et al. (2005), but this is not confirmed by the rotation properties. This shows that there is no general correlation between magnetic fields and the rotation rate of structures, at least in higher levels of solar atmosphere.

\section{Discussion}

In this section we discuss the problem of RR variation during the cycle and the long-term variation of RR of the full cycles. At the end is given a short summary of our essential findings.

The sunspots are scattered on the solar disk between $\pm 35^{\circ}$ latitude, so that at the same time spots can show very different RR (Gilman \& Howard 1984; Pulkkien \& Tuominen 1998). Mouradian et al. (2002) show that the number of peaks of the power spectrum grows in accordance with cycle evolution. The changes of RR during the cycle are not systematic, as the differential rotation rule can suggest. To test the randomness hypothesis of spot scattering we carried out a superimposed epoch analysis by averaging the RR of the 14 cycles, with respect to the number of two-year sequences. Consequently, the observed variations in Fig. 3 are due to some irregular movements, witch is the "noise" level mentioned above (Sect. 5.1.3).

A slight variation of RR during the cycle is expected due to the movement of sunspots towards the equator. This movement is approximately linear for the average latitude and using the empirical law of differential rotation, we find that the rotation rate $\Omega$ variation with time $(t)$ is:

$\Omega(t)=A-B \sin ^{2}\left(\theta_{\mathrm{b}}-\frac{\theta_{\mathrm{b}}-\theta_{\mathrm{e}}}{T} t\right)$

where $A$ and $B$ are the parameters of the differential rule; $\theta_{\mathrm{b}}$ and $\theta_{\mathrm{e}}$ the average latitude of spots at the beginning and the end of the cycle; $T$ is duration of the cycle. $\Omega$ is independent of the sunspot number. With the usual values of $A$ and $B$, we find that $\Omega$ is a slightly increasing function of time and can reach only $0.5 \% \mathrm{~d}(\sim 3 \%)$. In the case of the use of another function for the average latitude variation of spots, the extreme values are still valid.

The important result of the present paper is the variation of solar activity rotation. The series of three slopes (Sect. 5.1.2) must be considered as a first approximation for 52.4 y periodicity. In Fig. 5 the 15 solar cycles (1849-2006) clearly show the the $52.4 \mathrm{y}$ periodic variation of RR, even during the noisy cycles 9 to 13. Besides the periodic variation, the slope is present along cycles, which can be the signature of oscillation due to a lower frequency.

Some other investigations treat the long time evolution of the solar activity cycles. In order to find a mechanism for solar cycle, Yoshimura (1979) recovered the idea foreseen by Wolf in 1859 about the periodicity of 5 times 11 year-cycles of sunspot number maxima. He calls the set of five successive cycles the "Grand Cycle". The Grand Cycle V composed of cycles 14 to 20 shows quite good correspondence with the minimum of the $52 \mathrm{y}$ function of the present paper, but the maximum is slightly shifted. The Grand Cycle IV (cycles 11 to 15 ) shows an irregular behaviour, so that it was qualified as an "anomalous Grand Cycle". Consequently, in the limits of our results, any positive conclusion cannot be drawn concerning a possible relation between sunspot number and magnetic flux rotation. Concerning this possible relation, we have some doubt because cycle 19 is a counterexample: the strongest cycle in the history of sunspot numbers has a weak and slow variation of RR (Fig. 3).

Ikhsanov \& Vitinskii (1980) consider the synodic rotation rate of equatorial sunspot groups by analysing data of the Greenwich Photoheliograph Results from 1875 to 1954. They found similar rotation rate variation in agreement with our results (Sect. 5.2), and note that "the period of variation is five 11 -year cycles and the maximum amplitude is $\pm 0.2^{\circ} / \mathrm{d} / \mathrm{y}$ ". These figures are identical to those above given the limits i.e. 51.1$54.7 \mathrm{y}$ and $0.20-0.32^{\circ} / \mathrm{d} / \mathrm{y}$, the same results from two different data sets and different computing techniques.

Using the Greenwich Photoheliograph Results for the period 1874 to 1981 Brajša et al. (2006), measured the position of the sunspot group between latitudes $\pm 30^{\circ}$ and applied the residual method to measure the RR. Brajša et al. computed the power spectrum frequency of rotation periods and found a periodicity of $32.6 \mathrm{y}$, at the limit of the spectrum. Javaraih et al. (2005) determined the long term variation of sunspot differential rotation parameters. They obtained a continuum secular variation for $A$ 
and $B$ coefficients of the differential rotation rule. The short communication of Eddy et al. (1978) notes a secular variation of RR.

Two hypotheses can be suggested to explain the causes of time variation of solar activity rotation rate with a period of about 52 years:

- Variation of differential rotation shape from cycle to cycle may have very little impact on the global rotation rate (Javaraiah et al. 2005; Thompson et al. 2003).

- The hypothesis of continuous distortion of the solar spheroid by prolate-oblate oscillation, producing accelerationdeceleration of higher layers of the sun, seems a good candidate for the 52-year oscillation. This hypothesis can be tested by the ratio of polar to equatorial solar diameters.

Results can be summarized as follows:

- The solar activity rotation rate is not a constant figure.

- The Carrington rotation rate is a constant value, whereas the global rotation is rather a parameter, as show by the dotted line in Fig. 5.

- Sunspots and coronal activity have the same rotation rate, implying the quasi-rigid rotation of solar activity.

- Asymmetry of sunspot numbers between northern and the nouthern hemispheres is not confirmed by the rotation rate asymmetry.

Acknowledgements. The authors acknowledge the SIDC-team of the Data Centre of the Sunspot Index from the Royal Observatory of Belgium for providing sunspot daily data. This research used the facilities of the Canadian Astronomy Data Centre operated by the National Research Council of Canada with the support of the Canadian Space Agency, for coronal radio flux data. We also thank the teams of the Institute of Physics, University of Graz (Austria) and the Astronomical Institute Tatranská Lomnica (Slovakia), whose data of solar sunspot number per hemisphere are used in this paper. The authors thank Drs J. Leibacher (NSO Tucson) and D. Gambis (Observatoire de Paris) for instructive discussions. The authors also are thankful to the referee for valuable remarks and suggestions.

\section{References}

Ballester, J. L., Oliver, R., \& Carbonell, M. 2005, A\&A, 431, L5

Balthasar, H. 2007, A\&A, 471, 281

Beck, J. G. 1999, Sol. Phys., 191, 47

Berdyugina, S. V., \& Usoskin, I. G. 2003, A\&A, 405, 1121

Brajša, R., Ruždjak, D., \& Wöhl, H. 2006, Sol. Phys., 237, 365

Castenmiller, M. J. M., Zwaan, C., \& van der Zalm, E. B. J. 1986, Sol. Phys., 105,237

Eddy, J. A., Noyes, R. W., Wolbach, J. G., et al. 1978, BAAS, 10, 400

Gaizauskas, V., Harvey, K. L., Harvey, J. W., \& Zwaan, C. 1983, ApJ, 265, 1056

Gilman, P. A., \& Howard, R. 1984, ApJ, 283, 385

Graf, W. 1974, Sol. Phys., 37, 257

Howard, R. 1984, ARA\&A, 22, 131

Hoyt, D. V., \& Schatten, K. H. 1998, Sol. Phys., 181, 491

Ikhsanov, R. N., \& Vitinskii, Yu, I. 1980, Sov. Phys. Dokl., 25(9), 664

Javaraiah, J., Bertello, L., \& Ulrich, R. K. 2005, Sol. Phys., 232, 25

Juckett, D. A. 2000, Sol. Phys., 191, 201

Libbrecht, K. G., \& Morrow, C. A. 1991, in Solar Interior and Atmosphere, ed. A. N. Cox, W. C. Livingston, \& M. S. Matthews (The Univ. of Arizona Press), 479

Mouradian, Z. 2007, in Fifty Years of Romanian Astrophysics, ed. C. Dumitache,

N. A. Popescu, M. D. Suran, \& V. Mioc, AIP CP895, 59

Mouradian, Z., \& Heristchi, D. 2005, Hvar Obs. Bull., 29, 1

Mouradian, Z., Bocchia, R., \& Botton, C. 2000, C. R. Acad. Sci. Paris, Série IV, 1,353

Mouradian, Z., Bocchia, R., \& Botton, C. 2002, A\&A, 394, 1103

Newton, H. W., \& Nunn, M. L. 1951, MNRAS, 111, 413

Pojoga, S., \& Cudnik, B. 2002, Sol. Phys., 208, 17

Pulkkien, P., \& Tuominen, I. 1998, ApJ, 322, 748

Schröter, H. E. 1985, Sol. Phys., 100, 141

Snodgrass, H. B. 1992, in The Solar Cycle, ed. K. L. Harvey, ASP Conf. Ser., 27,205

Temmer, M., Rybak, K., Bendk, P., et al. 2006, A\&A, 447, 735

Thompson, M. J., Christensen-Dalsgaard, J., Miesch, M. S., \& Toomre, J. 2003, ARA\&A, 41, 599

Trellis, M. 1971, C. R. Acad. Sc. Paris, 272, Serie B, 549

Usoskin, I. G., Berdyugina, S. V., Moss, D., \& Sokoloff, D. D. 2007, Adv. Space Res., 40, 951

Wittmann, A. D. 1996, Sol. Phys., 168, 211

Yoshimura, H. 1979, ApJ, 227, 1047 